\title{
Correction to: Geniposide Suppresses Interleukin-1 $\beta$-Induced Inflammation and Apoptosis in Rat Chondrocytes via the PI3K/Akt/NF-kB Signaling Pathway
}

\author{
Tianlong Pan, ${ }^{1}$ Xuchao Shi, ${ }^{1}$ Huan Chen, ${ }^{2}$ Rong Chen, ${ }^{1}$ Dengying Wu, ${ }^{1}$ Zeng Lin, ${ }^{1}$ \\ Jingdong Zhang, ${ }^{1}$ and Jun Pan ${ }^{1,3}$
}

Correction to: Inflammation (2018) 41(2): 390-399

https://doi.org/10.1007/s10753-017-0694-2

The original version of this article contained mistakes, and the authors would like to correct them. The correct details are given below:

- Fig. 4 A. In the GPO group, the S-O and H-E staining pictures were used incorrectly. This is our mistake.

- Fig. 4 B. The name of the GPO drug treatment group is wrong, and the correct name should be OA+GPO.

The correct figure 4 can be seen at the next page.

The authors apologize for potential issues raised by these mistakes.

The online version of the original article can be found at https://doi.org/ 10.1007/s10753-017-0694-2

\footnotetext{
${ }^{1}$ Department of Orthopaedics, The Second Affiliated Hospital and Yuying Children's Hospital of Wenzhou Medical University, 109 Xue Yuan Xi Road, Wenzhou, Zhejiang, 325000, China

${ }^{2}$ Department of Orthopaedics, Yongjia County People's Hospital, 37 Yong Zhong Road, Shang Tang Town, Yongjia County, Zhejiang, 325100, China

${ }^{3}$ To whom correspondence should be addressed at Department of Orthopaedics, The Second Affiliated Hospital and Yuying Children's Hospital of Wenzhou Medical University, 109 Xue Yuan Xi Road, Wenzhou, Zhejiang, 325000, China. E-mail: panjun@wmu.edu.cn
} 

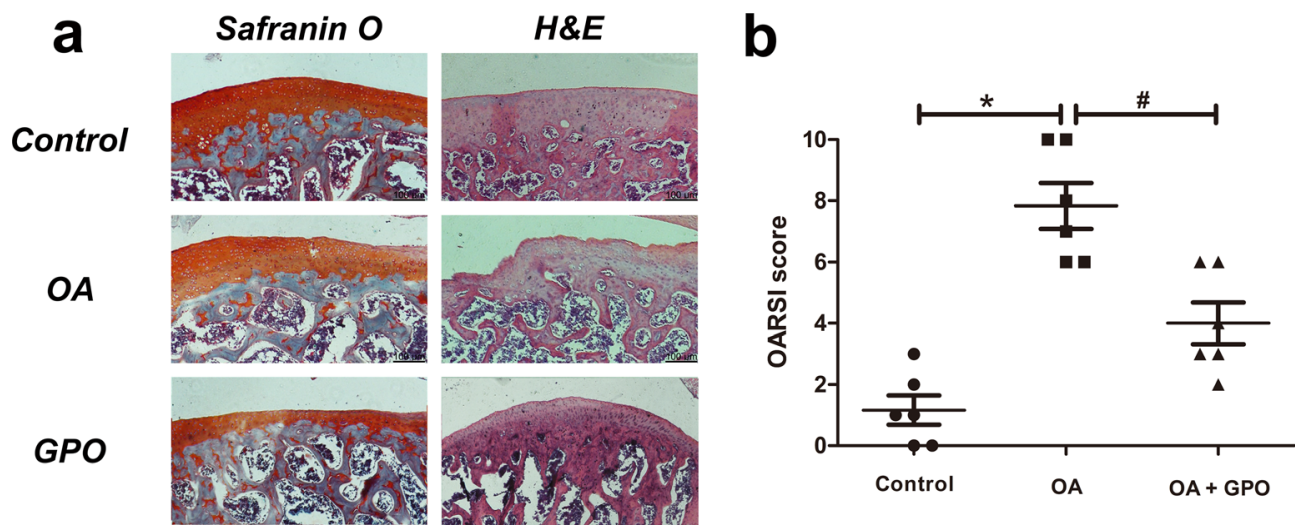

Fig. 4. The addition of GPO reduced the cartilage destruction in the OA ratmodel, assayed by Safranin-O staining, as well as H\&E staining (a). Rat received intraperitoneal injections of GPO or vehicle (DMSO) during the fourth week after surgery. The OARSI score was calculated for each group (b). Values are mean \pm SD. Significant differences from the control group are indicated by $* P<0.05$. Significant differences from the OA group are indicated by \# $P<0.05$. 